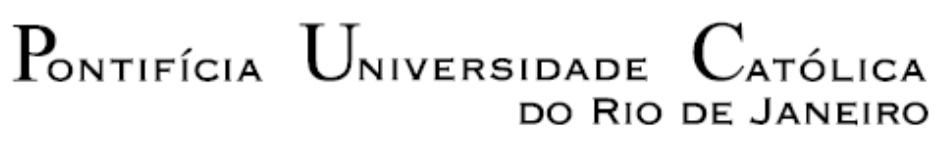

Maurício Gruzman

Sistema de Acompanhamento de Alvos Montado em um Corpo em Movimento

Tese apresentada ao Programa de Pós-Graduação em Engenharia Mecânica da PUC-Rio como requisito parcial para obtenção do título de Doutor em Engenharia Mecânica.

Orientador: Prof. Hans Ingo Weber Co-Orientador: Prof. Luciano Luporini Menegaldo 


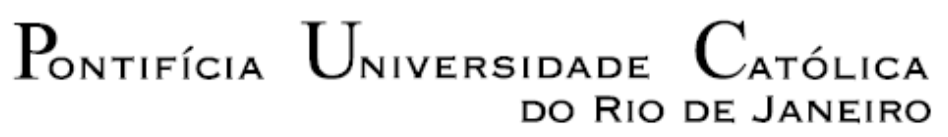

Maurício Gruzman

\section{Sistema de Acompanhamento de Alvos Montado em um \\ Corpo em Movimento}

Tese apresentada como requisito parcial para obtenção do grau de Doutor pelo Programa de Pós-Graduação em Engenharia Mecânica do Centro Técnico Científico da PUC-Rio. Aprovada pela Comissão Examinadora abaixo assinada.

Prof. Hans Ingo Weber Orientador Departamento de Engenharia Mecânica - PUC-Rio

Prof. Luciano Luporini Menegaldo Co-Orientador Instituto Militar de Engenharia

Prof. Arthur Martins Barbosa Braga Departamento de Engenharia Mecânica - PUC-Rio

Prof. Agenor de Toledo Fleury Centro Universitário da FEI

Prof. Ricardo Teixeira da Costa Neto Instituto Militar de Engenharia

Dr. Pedro Cunha Campos Roquette Instituto de Pesquisas da Marinha

Prof. José Eugenio Leal

Coordenador Setorial do Centro Técnico Científico - PUC-Rio

Rio de Janeiro, 12 de abril de 2011 
Todos os direitos reservados. É proibida a reprodução total ou parcial do trabalho sem autorização da universidade, do autor e do orientador.

\section{Maurício Gruzman}

Graduou-se em Engenharia Mecânica na Universidade Federal do Rio de Janeiro em 1998. Concluiu o Curso de Mestrado no Instituto Militar de Engenharia em 2003 onde elaborou uma dissertação na área de dinâmica de sistemas multi-corpos rígidos. Durante os estudos de Doutorado publicou junto com seu orientador e co-orientador um trabalho em revista internacional (Mathematical Problems in Engineering - Vol.2010), dois capítulos de livro (Proceedings of the $15^{\text {th }}$ International Workshop on Dynamics and Control e ABCM Symposium Series in Mechatronics - Vol.4) e apresentou vários trabalhos em congressos nacionais $\mathrm{e}$ internacionais (DINAME, CILAMCE, COBEM e CONEM).

Ficha Catalográfica

Gruzman, Maurício

Sistema de acompanhamento de alvos montado em um corpo em movimento / Maurício Gruzman ; orientador: Hans Ingo Weber ; co-orientador: Luciano Luporini Menegaldo. - 2011.

170 f. : il.(color.) ; $30 \mathrm{~cm}$

Tese (doutorado)-Pontifícia Universidade Católica do Rio de Janeiro, Departamento de Engenharia Mecânica, 2011.

Inclui bibliografia

1. Engenharia mecânica - Teses. 2. Sistemas de acompanhamento de alvos. 3. Dinâmica de sistemas multi-corpos rígidos. 4. Controle. 5. Folga. 6. Atrito. I. Weber, Hans Ingo. II. Menegaldo, Luciano Luporini. III. Pontifícia Universidade Católica do Rio de Janeiro. Departamento de Engenharia Mecânica. IV. Título. 


\section{Agradecimentos}

Gostaria de agradecer a todas as pessoas que tornaram possível a elaboração deste trabalho, em especial:

Aos professores Hans Ingo Weber e Luciano Luporini Menegaldo pela confiança em mim depositada, orientação e todos os conhecimentos transmitidos.

A meu filho Guilherme que, apesar de sua pouca idade, sempre compreendeu a importância deste trabalho para mim.

A minha esposa, aos meus pais, irmãos e toda a minha família pelo apoio dado durante este curso de Doutorado.

Aos professores membros da banca, pelos comentários e sugestões feitas.

Aos professores do Departamento de Engenharia Mecânica da PUC-Rio, pelos momentos compartilhados e conselhos a nível acadêmico.

Aos colegas do Laboratório de Dinâmica e Vibrações do Departamento de Engenharia Mecânica da PUC-Rio, por todo o apoio e pelos momentos gratos.

Ao colega Wagner Epifânio da Cruz do Laboratório de Dinâmica e Vibrações da PUC-Rio pelo apoio na montagem da bancada de testes.

Ao professor Ney Bruno do Instituto Militar de Engenharia e ao engenheiro Leibich Gruzman, meu pai, pelo apoio na montagem dos circuitos eletrônicos utilizados.

Ao $\mathrm{CNPq}$ e à PUC-Rio, pelos auxílios concedidos, sem os quais este trabalho não poderia ter sido realizado. 


\section{Resumo}

Gruzman, Maurício; Weber, Hans; Luporini Menegaldo, Luciano. Sistema de Acompanhamento de Alvos Montado em um Corpo em Movimento. Rio de Janeiro, 2011. 170p. Tese de Doutorado - Departamento de Engenharia Mecânica, Pontifícia Universidade Católica do Rio de Janeiro.

Neste trabalho estuda-se um sistema de acompanhamento de alvos, também conhecido como sistema de rastreamento de alvos, do tipo "pan-tilt" atuado por motores de corrente contínua e fixo em um corpo em movimento. Para tanto é montada uma bancada de testes e implementa-se um programa de simulação. A modelagem para o programa é feita no domínio do tempo, permitindo a utilização de equações bastante complexas para representar o sistema, o que não é possível quando se utiliza modelos no domínio da freqüência. Apesar de se modelar o sistema com corpos rígidos, flexibilidades e amortecimentos estruturais devido aos redutores de velocidade são considerados. Erros nos sensores, folgas nos redutores, atritos seco e viscoso, limites de saturação para as correntes e tensões nas armaduras dos motores também são considerados. Um método para a inclusão dos atrasos de tempo para atualização dos sinais de controle e dados obtidos pelos sensores durante a integração numérica das equações de movimento é apresentado. Para controlar o sistema utilizam-se controladores que não requerem o modelo matemático da planta, tanto na bancada de testes como no programa de simulação. Três tipos diferentes de arquitetura de controle são propostas, chamadas neste trabalho de tipo 1, tipo 2 e tipo 3. A complexidade delas aumenta à medida que mais sensores estão disponíveis no sistema. A arquitetura do tipo 1 destina-se a sistemas onde se possui apenas sensores que fornecem os erros angulares de azimute e elevação do alvo. Se, além deste sensor, também houver sensores para medir as posições angulares relativas entre os elos do mecanismo usa-se a arquitetura do tipo 2. Se houver, ainda, sensores de velocidades angulares inerciais pode-se utilizar a arquitetura do tipo 3. Por fim são apresentados resultados de experimentos e simulações onde se compara o desempenho do sistema com cada tipo de arquitetura de controle.

\section{Palavras - chave}

Sistemas de Acompanhamento de Alvos; Dinâmica de Sistemas MultiCorpos Rígidos; Controle; Folga; Atrito; Atrasos de Tempo no Controle. 


\section{Abstract}

Gruzman, Maurício; Weber, Hans(Advisor); Luporini Menegaldo, Luciano(Co-advisor). Target Tracking System Mounted in a Moving Body. Rio de Janeiro, 2011. 170p. Doctorate Thesis - Departamento de Engenharia Mecânica, Pontifícia Universidade Católica do Rio de Janeiro.

A study on a pan-tilt type target tracking system actuated by permanent magnet DC motors and assembled in a moving body is presented in this work. To achieve such objective, an experimental test bed is constructed and a simulation program is implemented. The mechanical model is derived and simulated in time domain. This approach allows using accurate non-linear equations to represent system behavior, otherwise infeasible in frequency domain. Although the system is modeled with rigid bodies, flexibility and structural damping due to the gearboxes are considered. Sensor errors, backlash in the gearboxes, dry and viscous friction, saturation limits for armature current and tension of the motors are also considered. A method to include the time delays for the control signal updates, as well as time delays due to sensor dynamic response, during the numerical integration of the equations of motion, is presented. Controllers that require no mathematical model of the plant are employed in the experimental test bed and in the simulation program. Three different control architectures are proposed, called in this work type 1, type 2 and type 3 . Their complexity increases depending on the number of available sensors. The type 1 is applied to systems with only one sensor that provides the targets angular azimuth and elevation errors. If, besides this sensor, sensors to measure the relative angular positions between the mechanism links are available type 2 architecture is used. In addition, if sensors to measure inertial angular speeds are also available, type 3 architecture can be used. Finally, experimental and numerical results, comparing system performance with each control architecture are presented.

\section{Keywords}

Target Tracking System; Rigid Multi-Body System Dynamics; Control; Backlash; Friction; Control Time Delays. 


\section{Sumário}

1. Introdução 29

1.1. Configurações do mecanismo 30

1.2. Objetivos 33

1.3. Revisão bibliográfica 34

1.4. Terminologia 37

1.5. Descrição da tese 39

2. Equações de movimento do sistema 40

2.1. Introdução 40

2.2. Lagrangeano do sistema 42

2.2.1. Energias cinéticas dos corpos 46

2.2.2. Energias potenciais dos corpos 49

2.3. Obtenção dos termos à esquerda nas equações de Lagrange 52

2.4. Torques generalizados não conservativos 52

2.4.1. Torque eletromotriz 53

2.4.2. Torques devido aos acoplamentos entre os corpos $R 1$ e 1 e $R 2$ e 2

2.4.3. Torques devido ao atrito viscoso 59

2.4.4. Torques devido ao atrito seco 59

2.5. Solução aproximada das equações de movimento 67

3. Controle 71

3.1. Introdução 71

3.2. Sistema apenas com sensor de visão 75

3.2.1. Arquitetura de controle 75

3.2.2. Controladores utilizados 76

3.2.2.1. Ajuste dos ganhos dos controladores 78

3.3. Sistema com sensor de visão e sensores para medir os ângulos $\alpha$ e $\beta \quad 80$

3.3.1. Arquitetura de controle 80 
3.3.2.1. Controladores utilizados nas malhas internas 86

3.3.2.2. Ajuste dos ganhos dos controladores utilizados nas malhas internas

3.4. Sistema com sensor de visão, girômetros e sensores para medir $\alpha$ e $\beta$

3.4.1. Arquitetura de controle 87

3.4.2. Malha interna (malha de estabilização) 90

3.4.2.1. Ajuste dos ganhos da malha interna 90

3.4.3. Malha externa (malha de perseguição) 91

3.4.3.1. Ajuste dos ganhos utilizados para o controlador da malha externa

3.4.4. Arquitetura completa com malhas internas e externas detalhadas

99

3.5. Erros nos sensores 100

$\begin{array}{ll}\text { 3.6. Considerações finais } & 103\end{array}$

4. Simulações 107

4.1. Introdução 107

4.2. Parâmetros utilizados nas simulações 107

4.2.1. Sistema de alimentação dos motores elétricos 107

$\begin{array}{ll}\text { 4.2.2. Corpo } 1 & 108\end{array}$

4.2.3. Corpo $2 \quad 108$

$\begin{array}{ll}\text { 4.2.4. Motor } 1 & 108\end{array}$

4.2.5. Motor $2 \quad 109$

4.2.6. Redutor entre o rotor 1 e o corpo 1

4.2.7. Redutor entre o rotor 2 e o corpo $2 \quad 110$

4.2.8. Sensores de posição angular relativa e sensor de visão 110

4.2.9. Outras informações 111

4.3. Simulações 111

4.3.1. Situação 1: Corpo 0 e alvo fixos 111

4.3.2. Situação 2: Corpo 0 fixo e alvo movendo-se 112

4.3.3. Situação 3: Corpo 0 movendo-se e alvo fixo 113 
4.3.4. Situação 4: Corpo 0 e alvo movendo-se 114

4.4. Resultados das simulações 115

4.4.1. Arquitetura de controle do tipo 1

4.4.1.1. Situação $1 \quad 115$

4.4.1.2. Situação $2 \quad 119$

4.4.1.3. Situação $3 \quad 120$

4.4.1.4. Situação $4 \quad 122$

4.4.2. Arquitetura de controle do tipo 2

4.4.2.1. Situação 1

4.4.2.2. Situação $2 \quad 126$

4.4.2.3. Situação $3 \quad 128$

4.4.2.4. Situação $4 \quad 128$

4.4.3. Arquitetura de controle do tipo $3 \quad 129$

4.4.3.1. Situação $1 \quad 129$

4.4.3.2. Situação $2 \quad 130$

4.4.3.3. Situação $3 \quad 131$

4.4.3.4. Situação 4

4.4.4. Influência dos erros aleatórios nos sensores 132

4.4.4.1. Arquitetura de controle do tipo 1

4.4.4.2. Arquitetura de controle do tipo 2

4.4.4.3. Arquitetura de controle do tipo $3 \quad 134$

4.4.4.3.1. Erros de medição apenas no sensor de visão 135

4.4.4.3.2. Erros de medição apenas nos girômetros 135

4.4.4.4. Comparação entre os controladores 136

$\begin{array}{ll}\text { 4.5. Análise de resultados } & 137\end{array}$

5. Bancada de testes 139

5.1. Introdução 139

5.2. Componentes da bancada de testes 141

5.2.1. Alvo 141

5.2.2. Câmera digital 142

5.2.3. Encoders e tacômetros 142

$\begin{array}{ll}\text { 5.2.4. Placa multifuncional } & 143\end{array}$ 
5.2.5. Motores/redutores do sistema de acompanhamento de alvos

5.2.6. Amplificadores de potência 144

5.2.7. Computador 144

5.2.8. Programa em Labview ${ }^{\circledR} \quad 144$

5.2.8.1. Arquitetura de controle do tipo 1

5.2.8.1.1. Ajustes do sensor de visão 145

5.2.8.1.2. Ajustes dos ganhos dos controladores PID 149

5.2.8.1.3. Delta T 150

5.2.8.1.4. Ligar motores $\quad 150$

5.2.8.1.5. Dados de saída $\quad 150$

5.2.8.2. Arquitetura de controle do tipo 2

5.2.9. Base 151

5.3. Testes 152

5.3.1. Introdução 152

5.3.2. Arquitetura de controle do tipo 1

5.3.2.1. Teste 1 - alvo fixo e corpo 0 fixo 153

5.3.2.2. Teste 2 - alvo móvel e corpo 0 fixo 155

5.3.2.3. Teste 3 - alvo fixo, corpo 0 fixo e ganhos dos controladores ajustados por Ziegler-Nichols 156

5.3.3. Arquitetura de controle do tipo 2

5.3.3.1. Teste 4 - alvo fixo, corpo 0 fixo e ganhos dos controladores ajustados por Ziegler-Nichols 157

5.4. Análise de resultados 158

6. Conclusões e sugestões para trabalhos futuros 159

6.1. Conclusões 159

6.2. Sugestões para trabalhos futuros 163

7. Referências bibliográficas 


\section{Lista de Figuras}

Figura 1.1 - Mecanismo com três graus de liberdade 31

Figura 1.2 - Mecanismo com dois graus de liberdade 31

Figura 1.3 - Mecanismo de 2 graus de liberdade e atuadores na base (Antolovic [1]) 32

Figura 1.4 - Mecanismo de 3 graus de liberdade e atuadores na base (Wagner et al [2]) 32

Figura 1.5 - Mecanismo de 3 graus de liberdade e atuadores na base (Bang et al [3]) 32

Figura 1.6 - Mecanismo com dois graus de liberdade e 2 atuadores

Figura 2.1 - Corpos que compõem o sistema 41

Figura 2.2 - Sistemas de coordenadas utilizados 43

Figura 2.3 - Vetores posição 44

Figura 2.4 - Rotação entre os sistemas de coordenadas 44

Figura 2.5 - Orientação do corpo $0 \quad 45$

Figura 2.6 - Plano de referência para cômputo da energia potencial 49

Figura 2.7 - Sistema em relação ao plano de referência 50

Figura 2.8 - Motor C.C. de imã permanente controlado pela tensão na armadura 53

Figura 2.9 - Algoritmo para se incluir o efeito de saturação na corrente da armadura $\quad 55$

Figura 2.10 - Modelo adotado para representar o redutor 57

Figura 2.11 - Pontos de contato 57

Figura 2.12 - Torque de atrito utilizado dependendo da velocidade angular 61

Figura 2.13 - Velocidade angular residual $\quad 62$

Figura 2.14 - Torque devido ao atrito seco 65

Figura 2.15 - Atrito seco nos motores elétricos 66 
Figura 3.1 - Erros angulares e imagem capturada pelo sensor de visão

Figura 3.2 - Campo de visão do sensor 73

Figura 3.3 - Vetor posição do alvo com respeito ao ponto $b \quad 73$

Figura 3.4 - Obtenção do vetor posição do alvo com respeito ao ponto $b$ durante a simulação $\quad 74$

Figura 3.5 - Arquitetura de controle do tipo $1 \quad 76$

Figura 3.6 - Controlador proporcional genérico 78

Figura 3.7 - Ganho e período críticos 79

Figura 3.8 - Sistema parado antes de se iniciar o procedimento de Ziegler-Nichols $\quad 79$

Figura 3.9 - Sistema com sensor de visão e encoders 80

Figura 3.10 - Arquitetura de controle do tipo $2 \quad 81$

Figura 3.11- Atrasos das malhas que devem ser considerados na integração numérica 81

Figura 3.12 - Entradas para o cálculo de $\alpha_{d}$ e $\beta_{d}$

Figura 3.13 - Componentes do vetor $\mathbf{n}$ no sistema de coordenadas solidário ao corpo 1

Figura 3.14 - Sistemas de coordenadas 1' e 2' 84

Figura 3.15 - Sensor com girômetros montado no corpo $2 \quad 87$

Figura 3.16 - Arquitetura de controle do tipo 3 em linhas gerais 88

Figura 3.17 - Blocos de um controlador de lógica fuzzy 91

Figura 3.18 - Entradas e saída do controlador fuzzy utilizado $\quad 92$

Figura 3.19 - Funções de pertinência 93

Figura 3.20 - Utilização de ganhos para se evitar alterações nas funções de pertinência. 93

Figura 3.21 - Funções de pertinência normalizadas 94

Figura 3.22 - Superfície de controle 94

Figura 3.23 - Funções de pertinência de NiS 95

Figura 3.24 - Representação esquemática da aproximação para a derivada do erro $\quad 97$

Figura 3.25 - Visão do alvo em três instantes 98 
Figura 3.26 - Detalhamento da arquitetura de controle do tipo 3 completa

Figura 3.27 - Curva de probabilidade de uma variável aleatória uniforme

Figura 3.28 - Curva de probabilidade de uma variável aleatória gaussiana

Figura 3.29 - Encoder ótico incremental com 8 fendas

Figura 3.30 - Ângulos verdadeiros (curvas azuis) e ângulos medidos (curvas vermelhas)

Figura 4.1 - Alvo e corpo 0 fixos

Figura 4.2 - Movimento do alvo 112

Figura 4.3 - Alvo fixo, corpo 0 em movimento 113

Figura 4.4 - Alvo e corpo 0 em movimento 114

Figura 4.5 - Simulação 1:Erros angulares de azimute e elevação em função do tempo

Figura 4.6 - Simulação 2:Erros angulares de azimute e elevação em função do tempo

Figura 4.7 - Simulação 3:Erros angulares de azimute e elevação em função do tempo

Figura 4.8 - Simulação 4:Erros angulares de azimute e elevação em função do tempo

Figura 4.9 - Simulação 5:Erros angulares de azimute e elevação em função do tempo

Figura 4.10 - Simulação 6:Erros angulares de azimute e elevação em função do tempo

Figura 4.11 - Simulação 7:Erros angulares de azimute e elevação em função do tempo

Figura 4.12 - Simulação 8:Erros angulares de azimute e elevação em função do tempo

Figura 4.13 - Simulação 9:Erros angulares de azimute e elevação em função do tempo

Figura 4.14 - Simulação 10:Erros angulares de azimute e elevação em função do tempo 
Figura 4.15 - Simulação 11:Erros angulares de azimute e elevação em função do tempo

Figura 4.16 - Simulação 12:Erros angulares de azimute e elevação em função do tempo

Figura 4.17 - Simulação 13:Erros angulares de azimute e elevação em função do tempo

Figura 4.18 - Simulações 14 e 15:Erros angulares de azimute e elevação em função do tempo

Figura 4.19 - Simulação 16:Erros angulares de azimute e elevação em função do tempo

Figura 4.20 - Simulação 17:Erros angulares de azimute e elevação em função do tempo

Figura 4.21 - Simulação 18:Erros angulares de azimute e elevação em função do tempo

Figura 4.22 - Simulação 19:Erros angulares de azimute e elevação em função do tempo

Figura 4.23 - Simulações 20 e 21:Erros angulares de azimute e elevação em função do tempo

Figura 4.24 - Simulações 22 e 23:Erros angulares de azimute e elevação em função do tempo

Figura 4.25 - Simulação 24:Erros angulares de azimute e elevação em função do tempo

Figura 4.26 - Simulação 25:Erros angulares de azimute e elevação em função do tempo

Figura 4.27 - Simulação 26:Erros angulares de azimute e elevação em função do tempo

Figura 4.28 - Simulação 27:Erros angulares de azimute e elevação em função do tempo

Figura 4.29 - Simulação 28:Erros angulares de azimute e elevação em função do tempo

Figura 4.30 - Simulação 29:Erros angulares de azimute e elevação em função do tempo 
Figura 4.31 - Simulação 30:Erros angulares de azimute e elevação em função do tempo

Figura 4.32 - Simulação 31:Erros angulares de azimute e elevação em função do tempo

Figura 4.33 - Simulação 32:Erros angulares de azimute e elevação em função do tempo

Figura 4.34 - Simulação 33:Erros angulares de azimute e elevação em função do tempo

Figura 4.35 - Simulações 34, 35 e 36:Erros angulares de azimute e elevação em função do tempo

Figura 5.1 - Fotografia da bancada de testes, constituída pelo sistema de acompanhamento de alvos, base de acrílico e quadros de alumínio

Figura 5.2 - Fotografia da bancada de testes com ângulos de "pitch", "yaw" e "roll" diferentes de zero

Figura 5.3 - Fotografia do sistema de acompanhamento de alvos montado na base de acrílico

Figura 5.4 - Esquema experimental do sistema de acompanhamento de alvos

Figura 5.5 - Desenho esquemático do dispositivo para movimentação do alvo

Figura 5.6 - Fotografia do alvo preto fixo no disco branco à frente da cartolina branca

Figura 5.7 - Fotografia do conjunto encoder/tacômetro conectado ao corpo 1

Figura 5.8 - Amplificador de potência, fonte CC, motor CC e placa multifuncional

Figura 5.9 - Painel frontal do programa implementado em Labview $^{\circledR}$

Figura 5.10 - Conversão de pixels para radianos

Figura 5.11 - Conversão de pixels para radianos com compensação de distorção 
Figura 5.12 - Variação da relação pixels/rad em função das coordenadas do centróide

Figura 5.13 - Inclinação (a) e ponto onde a reta cruza o eixo vertical (b)

Figura 5.14 - Desenho esquemático do conjunto de quadros

Figura 5.15 - Desenho esquemático do motor do quadro externo

Figura 5.16 - Ângulos $\alpha$ (gráfico da esquerda) e $\beta$ (gráfico da direita) obtidos no teste 1

Figura 5.17 - Ângulos $\alpha$ (gráfico da esquerda) e $\beta$ (gráfico da direita) obtidos na simulação relativa ao teste 1

Figura 5.18 - Sobreposição dos resultados numéricos (em azul) e experimentais (em vermelho).

Figura 5.19 - Ângulos $\alpha$ (gráfico da esquerda) e $\beta$ (gráfico da direita) obtidos no teste 2

Figura 5.20 - Ângulos $\alpha$ (gráfico da esquerda) e $\beta$ (gráfico da direita) obtidos na simulação relativa ao teste 2

Figura 5.21 - Sobreposição dos resultados numéricos (em azul) e experimentais (em vermelho)

Figura 5.22 - Erros angulares de azimute (gráfico da esquerda) e elevação (gráfico da direita) obtidos no teste 3.

Figura 5.23 - Erros angulares de azimute (gráfico da esquerda) e elevação (gráfico da direita) obtidos no teste 4. 


\section{Lista de Tabelas}

Tabela 3.1. Ganhos do controlador PID (ou PI ou P) em função de $k_{\text {crit }}$ e $T_{\text {crit }}$

Tabela 3.2. Base de regras para o FLC com duas entradas $(\hat{\boldsymbol{e}}, \dot{\hat{\boldsymbol{e}}}) \quad 93$

Tabela 3.3. Base de regras para o FLC com três entradas (entrada 1, entrada 2, NiS)

Tabela 4.1. Ganhos do controlador PID (ou PI ou P) 117

Tabela 4.2. Ganhos do controlador PID (ou PI ou P) 123

Tabela 4.3. Ganhos do controlador PID (ou PI ou P) 125

Tabela 5.1. Coordenada $X_{\text {cen }}$ do centróide para diferentes posições do alvo

Tabela 5.2. Coordenada $Z_{\text {cen }}$ do centróide para diferentes posições do alvo 


\section{Lista de Símbolos}

\section{Símbolos Romanos}

$a$

Origem do sistema de coordenadas solidário ao corpo 0 .

$a(a z) \quad a(e l)$

Inclinações das retas obtidas pelo método dos mínimos quadrados (retas utilizadas para ajuste do número de pixels por radiano em função do número de pixels).

atan

Arco tangente.

$b$

Ponto de interseção dos eixos de elevação, de rotação e central.

$b(a z) \quad b(e l) \quad$ Pontos onde as retas obtidas pelo método dos mínimos quadrados (retas para ajuste do número de pixels por radiano em função do número de pixels) interceptam o eixo vertical.

$c$

$c_{1}$

$c_{2}$

C.C.

Origem do sistema de coordenadas solidário ao corpo 1 .

Coeficiente de atrito viscoso no corpo 1 .

Coeficiente de atrito viscoso no corpo 2.

Corrente contínua.

$c_{\text {numérico, } i}$

$\cos$

$c_{R 1}$

$c_{R 2}$

$c_{S}$

Coeficiente utilizado no termo de amortecimento numérico para um corpo $i$.

Cosseno.

Coeficiente de atrito viscoso no rotor $R 1$.

Coeficiente de atrito viscoso no rotor $R 2$.

Coeficiente de amortecimento estrutural resultante do redutor.

$c_{s 1} \quad$ Coeficiente de amortecimento estrutural resultante do redutor utilizado entre o rotor $R l$ e o corpo 1 .

$c_{s 2} \quad$ Coeficiente de amortecimento estrutural resultante do redutor utilizado entre o rotor $R 2$ e o corpo 2 .

d $\quad$ Origem do sistema de coordenadas solidário ao corpo 2 .

$d_{x_{2}} d_{y_{2}} d_{z_{2}} \quad$ Componentes do vetor posição do alvo com respeito ao ponto $b$ escrito em coordenadas do sistema solidário ao corpo 2 .

$\mathbf{d}_{\mathbf{b}}$

Vetor posição de um ponto $b$ com respeito a um ponto $a$, quando não se especifica o sistema de coordenas no qual ele é escrito. 


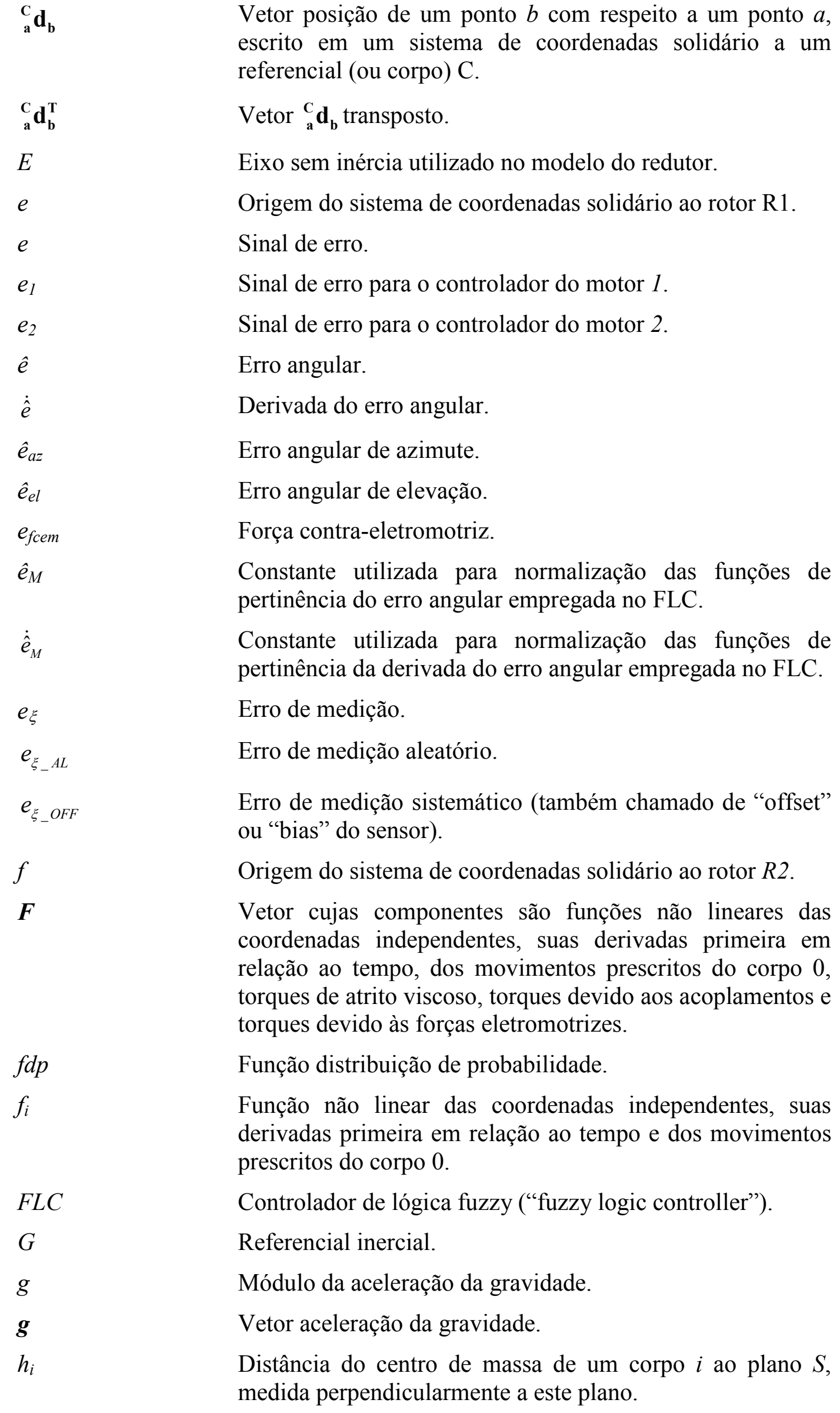

Vetor posição de um ponto $b$ com respeito a um ponto $a$, escrito em um sistema de coordenadas solidário a um referencial (ou corpo) C.

Vetor ${ }_{\mathbf{a}}^{\mathbf{C}} \mathbf{d}_{\mathbf{b}}$ transposto.

Eixo sem inércia utilizado no modelo do redutor.

Origem do sistema de coordenadas solidário ao rotor R1.

Sinal de erro.

Sinal de erro para o controlador do motor 1 .

Sinal de erro para o controlador do motor 2.

Erro angular.

Derivada do erro angular.

Erro angular de azimute.

Erro angular de elevação.

Força contra-eletromotriz.

Constante utilizada para normalização das funções de pertinência do erro angular empregada no FLC.

Constante utilizada para normalização das funções de pertinência da derivada do erro angular empregada no FLC.

Erro de medição.

Erro de medição aleatório.

Erro de medição sistemático (também chamado de "offset" ou "bias" do sensor).

Origem do sistema de coordenadas solidário ao rotor $R 2$.

Vetor cujas componentes são funções não lineares das coordenadas independentes, suas derivadas primeira em relação ao tempo, dos movimentos prescritos do corpo 0 , torques de atrito viscoso, torques devido aos acoplamentos e torques devido às forças eletromotrizes.

Função distribuição de probabilidade.

Função não linear das coordenadas independentes, suas derivadas primeira em relação ao tempo e dos movimentos prescritos do corpo 0 .

Controlador de lógica fuzzy ("fuzzy logic controller").

Referencial inercial.

Módulo da aceleração da gravidade.

Vetor aceleração da gravidade.

Distância do centro de massa de um corpo $i$ ao plano $S$, medida perpendicularmente a este plano. 
Corrente na armadura.

$i_{R 2}$

${ }^{A} I I_{A}$

$J_{i, j}$

$k$

K

$\mathbf{k}$

$K_{1}$

$K_{2}$

$k_{\text {crit }}$

$k_{d}$

$k_{d, R I}$

$k_{d, R 2}$

$k_{f c e m}$

$k_{f c e m, R I}$

$k_{f c e m, R 2}$

$k_{i}$

$k_{i, R 1}$

$k_{i, R 2}$

$k_{p}$

$k_{p, R l}$

$k_{p, R 2}$

$K_{R 1}$

$K_{R 2}$

$k_{s}$

$k_{s 1}$

$k_{s 2}$
Valor máximo da corrente na armadura.

Valor máximo da corrente na armadura do motor 1 .

Valor máximo da corrente na armadura do motor 2.

Corrente na armadura do motor 1 .

Corrente na armadura do motor 2 .

Tensor de inércia de um corpo rígido A escrito em coordenadas de um sistema solidário ao próprio corpo $\mathrm{A}$.

Função não linear das coordenadas independentes, suas derivadas primeira em relação ao tempo e dos movimentos prescritos do corpo 0 .

Instante de tempo discreto.

Energia cinética total do sistema.

Vetor unitário perpendicular ao plano S.

Energia cinética do corpo 1.

Energia cinética do corpo 2.

Ganho crítico observado durante o experimento de ZieglerNichols.

Ganho derivativo.

Ganho derivativo utilizado no controlador do motor 1 .

Ganho derivativo utilizado no controlador do motor 2.

Constante de força contra-eletromotriz.

Constante de força contra-eletromotriz do motor 1 .

Constante de força contra-eletromotriz do motor 2.

Ganho integral.

Ganho integral utilizado no controlador do motor 1 .

Ganho integral utilizado no controlador do motor 2.

Ganho proporcional.

Ganho proporcional utilizado no controlador do motor 1 .

Ganho proporcional utilizado no controlador do motor 2.

Energia cinética do rotor $R l$.

Energia cinética do rotor $R 2$.

Coeficiente de flexibilidade resultante do redutor.

Coeficiente de flexibilidade resultante do redutor utilizado entre o rotor R1 e o corpo 1 .

Coeficiente de flexibilidade resultante do redutor utilizado entre o rotor R2 e o corpo 2. 

$k_{\text {torque }}$
Constante de torque do motor.
$k_{\text {torque, } R I}$
Constante de torque do motor 1 .
$k_{\text {torque, } R 2}$
Constante de torque do motor 2 .
$l$
Distância do ponto b ao alvo ao longo do eixo central.
$\mathrm{L}$
Lagrangeano do sistema.
$\mathrm{L}_{1}$
Lagrangeano do corpo 1.
$\mathrm{L}_{2}$
Lagrangeano do corpo 2.
$\mathrm{L}_{\mathrm{R} 1}$
Lagrangeano do rotor $R l$.
$\mathrm{L}_{\mathrm{R} 2}$
Lagrangeano do rotor $R 2$.
$L$
Indutância da armadura.
$L_{R 1}$
Indutância da armadura do motor 1 .
$L_{R 2}$
Indutância da armadura do motor 2 .
$m_{1}$
Massa do corpo 1 .
M1 M2 M3
Ganhos utilizados no FLC.
$M 1_{R 1} M 2_{R 1} M 3_{R 1}$
Ganhos utilizados no FLC do controlador do motor 1.
$M 1_{R 2} M 2_{R 2} M 3_{R 2} \quad$ Ganhos utilizados no FLC do controlador do motor 2.
$m_{2}$
Massa do corpo 2.
$m_{R 1}$
Massa do rotor $R 1$.
$m_{R 2}$
Massa do rotor $R 2$.
n
Vetor unitário sob a linha que une o ponto $b$ ao alvo.
$\mathrm{N}$
Negativo.
$N$
Relação de redução no redutor.
$N_{l}$
Relação de redução no redutor acoplado ao motor 1 .
$\mathrm{N}_{2}$
Relação de redução no redutor acoplado ao motor 2.
$N_{F}$
Número de fendas no disco ótico do encoder.
NG
Negativo grande.
$\mathrm{NiS}$
Nível de saturação da tensão do motor.
$N i S_{R 1}$
Nível de saturação da tensão do motor 1 .
$\mathrm{Ni} \mathrm{S}_{R 2}$
Nível de saturação da tensão do motor 2 .
$\mathrm{NM}$
Negativo médio.
NP
Negativo pequeno.
$n_{x_{1}} n_{y_{1}} n_{z_{1}}$
Componentes do vetor $\mathbf{n}$ escrito em coordenadas do sistema solidário ao corpo 1 . 


\begin{tabular}{|c|c|}
\hline $\begin{array}{lll}n_{x_{1}^{\prime}} & n_{y_{1}^{\prime}} & n_{z_{1}^{\prime}}\end{array}$ & $\begin{array}{l}\text { Componentes do vetor } \mathbf{n} \text { escrito em coordenadas do sistema } \\
x^{\prime}{ }_{1} y^{\prime}{ }_{1} z_{1}^{\prime} \text {. }\end{array}$ \\
\hline$n_{x_{2}^{\prime}} \quad n_{y_{2}^{\prime}} \quad n_{z_{2}^{\prime}}$ & $\begin{array}{l}\text { Componentes do vetor } \mathbf{n} \text { escrito em coordenadas do sistema } \\
x_{2}^{\prime} y_{2}^{\prime} z_{2}^{\prime} \text {. }\end{array}$ \\
\hline $\mathcal{O}$ & $\begin{array}{l}\text { Origem do sistema de coordenadas solidário ao referencial } \\
\text { inercial. }\end{array}$ \\
\hline $\mathrm{P}$ & Positivo. \\
\hline$P$ & Resolução do encoder. \\
\hline PG & Positivo grande. \\
\hline$P I D$ & Proporcional-Integral-Derivativo. \\
\hline PM & Positivo médio. \\
\hline $\mathrm{PP}$ & Positivo pequeno. \\
\hline$P_{\alpha}$ & Resolução do encoder utilizado para medir o ângulo $\alpha$. \\
\hline$P_{\beta}$ & Resolução do encoder utilizado para medir o ângulo $\beta$. \\
\hline$p\left(e_{\xi_{-} A L}\right)$ & Probabilidade de uma variável aleatória. \\
\hline$q_{1} q_{2} q_{3} q_{4} q_{5} q_{6} q_{7} q_{8}$ & Coordenadas generalizadas do mecanismo. \\
\hline$R$ & Resistência da armadura. \\
\hline$r$ & Sinal de referência. \\
\hline$R 1$ & $\begin{array}{l}\text { Rotor } R 1 \text { (também chamado de corpo } R 1 \text {, rotor } 1 \text { ou rotor } \\
\text { do motor } 1 \text { ). }\end{array}$ \\
\hline$r_{1}$ & $\begin{array}{l}\text { Sinal de referência utilizado para ajustar os ganhos do } \\
\text { controlador do motor } 1 \text { pelo método de Ziegler-Nichols. }\end{array}$ \\
\hline$R 2$ & $\begin{array}{l}\text { Rotor } R 2 \text { (também chamado de corpo } R 2 \text {, rotor } 2 \text { ou rotor } \\
\text { do motor } 2 \text { ). }\end{array}$ \\
\hline$r_{2}$ & $\begin{array}{l}\text { Sinal de referência utilizado para ajustar os ganhos do } \\
\text { controlador do motor } 2 \text { pelo método de Ziegler-Nichols. }\end{array}$ \\
\hline$R_{R 1}$ & Resistência da armadura do motor 1 . \\
\hline$R_{R 2}$ & Resistência da armadura do motor 2 . \\
\hline$S$ & Plano de referência da energia potencial. \\
\hline$s$ & Saída da planta. \\
\hline $\operatorname{sen}$ & Seno. \\
\hline$t$ & Tempo. \\
\hline$t_{0}$ & Instante inicial de simulação. \\
\hline$t_{f}$ & Instante final de simulação. \\
\hline$T_{\text {acopl }}$ & Torque devido ao acoplamento entre dois corpos. \\
\hline
\end{tabular}



Torque devido ao acoplamento entre os corpos $l$ e $R l$, que atua no corpo 1 na direção $z_{1}$.
Torque devido ao acoplamento entre os corpos 2 e $R 2$, que atua no corpo 2 na direção $x_{2}$.
Torque devido ao acoplamento entre os corpos 1 e $R l$, que atua em $R 1$ na direção $z_{R l}$.
Torque devido ao acoplamento entre os corpos 2 e $R 2$, que atua em $R 2$ na direção $x_{R 2}$.
Torque devido ao acoplamento entre um rotor de um motor e uma carga, que atua na carga.
Torque devido ao acoplamento entre um rotor de um motor e uma carga, que atua no rotor.
Tangente.
Torque devido ao atrito seco.
Torque devido ao atrito seco no corpo 1 na direção $z_{1}$.
Torque devido ao atrito seco no corpo 2 na direção $x_{2}$.
Torque devido ao atrito seco em um corpo $i$.
Torque devido ao atrito seco no rotor $R 1$ na direção $z_{R 1}$.
Torque devido ao atrito seco no rotor $R 2$ na direção $x_{R 2}$.
Torque devido ao atrito viscoso.
Torque devido ao atrito viscoso no corpo 1 na direção $z_{1}$.
Torque devido ao atrito viscoso no corpo 2 na direção $x_{2}$.
Torque devido ao atrito viscoso no rotor $R 1$ na direção $z_{R 1}$.
Torque devido ao atrito viscoso no rotor $R 2$ na direção $x_{R 2}$.
Período crítico observado durante o experimento de Ziegler- Nichols.
Torque devido ao atrito seco dinâmico.
Torque devido ao atrito seco dinâmico no corpo 1 na direção $z_{1}$
Torque devido ao atrito seco dinâmico no corpo 2 na direção $x_{2}$
Torque devido ao atrito seco dinâmico em um corpo $i$.
Torque devido ao atrito seco dinâmico no rotor $R l$ na direção $z_{R 1}$.
Torque devido ao atrito seco dinâmico no rotor $R 2$ na direção $x_{R 2}$.

Torque no eixo sem inércia utilizado no modelo do redutor. 
$T_{E 1}$

$T_{E 2}$

$T_{\text {ele }}$

$T_{e l e, R I}$

$T_{\text {ele }, R 2}$

$T_{\max }$

$T_{\max , 1}$

$T_{\max , 2}$

$T_{\max , i}$

$T_{\max , R I}$

$T_{\max , R 2}$

$T_{\text {numérico }}$

$T_{\text {numérico, } i}$

$T_{\text {outros, } 1}$

$T_{\text {outros, } 2}$

$T_{\text {outros, } i}$

$T_{\text {outros, } R I}$

$T_{\text {outros,R2 }}$
Torque no eixo sem inércia utilizado no modelo do redutor localizado entre o rotor $R l$ e o corpo 1 .

Torque no eixo sem inércia utilizado no modelo do redutor localizado entre o rotor $R 2$ e o corpo 2 .

Torque eletromotriz.

Torque eletromotriz no rotor $R I$ na direção $z_{R l}$.

Torque eletromotriz no rotor $R 2$ na direção $x_{R 2}$.

Valor máximo do torque devido ao atrito seco estático.

Valor máximo do torque devido ao atrito seco estático no corpo 1 , na direção $z_{l}$.

Valor máximo do torque devido ao atrito seco estático no corpo 2, na direção $x_{2}$.

Valor máximo do torque devido ao atrito seco estático em um corpo $i$.

Valor máximo do torque devido ao atrito seco estático no rotor $R 1$, na direção $z_{R 1}$.

Valor máximo do torque devido ao atrito seco estático no rotor $R 2$, na direção $x_{R 2}$.

Termo de amortecimento numérico.

Termo de amortecimento numérico para um corpo $i$.

Somatório de todos os torques que atuam no corpo 1, na direção $z_{l}$, incluindo os torques inerciais decorrentes do movimento de outros corpos, mas excluindo os torques devido ao atrito seco.

Somatório de todos os torques que atuam no corpo 2, na direção $x_{2}$, incluindo os torques inerciais decorrentes do movimento de outros corpos, mas excluindo os torques devido ao atrito seco.

Somatório de todos os torques que atuam em um corpo $i$, incluindo os torques inerciais decorrentes do movimento de outros corpos, mas excluindo os torques devido ao atrito seco.

Somatório de todos os torques que atuam no rotor $R l$, na direção $z_{R l}$, incluindo os torques inerciais decorrentes do movimento de outros corpos, mas excluindo os torques devido ao atrito seco.

Somatório de todos os torques que atuam no rotor $R 2$, na direção $x_{R 2}$, incluindo os torques inerciais decorrentes do movimento de outros corpos, mas excluindo os torques devido ao atrito seco. 

${ }^{B} T^{A}$
Matriz de transformação de coordenadas de um sistema solidário ao referencial A para um sistema solidário ao referencial $\mathrm{B}$.
$U$
$U_{1}$
$U_{2}$
$U_{R 1}$
$U_{R 2}$
$u$
$u_{R 1}$
$u_{R 2}$
$U_{L, R I}$
$U_{L, R 2}$
${ }_{\mathbf{B}}^{\mathrm{C}} \mathbf{v}_{\mathbf{a}}$
${ }_{\mathbf{B}}^{\mathrm{C}} \mathbf{v}_{\mathrm{a}}^{\mathrm{T}}$
$\begin{array}{llll}x_{0} & y_{0} & z_{0}\end{array}$
$\begin{array}{lll}x_{1} & y_{1} & z_{1}\end{array}$
$\begin{array}{llll}x_{2} & y_{2} & z_{2}\end{array}$
$x_{1}^{\prime} y_{1}^{\prime}{ }_{1} z_{1}^{\prime}$
$x_{2}^{\prime} y_{2}^{\prime} z_{2}^{\prime}$
$\begin{array}{lll}x_{a} & y_{a} & z_{a}\end{array}$
$x_{\text {alvo }} y_{\text {alvo }} z_{\text {alvo }}$
$X_{\text {cen }} Z_{\text {cen }}$
$\begin{array}{llll}x_{G} & y_{G} & z_{G}\end{array}$
$\begin{array}{lll}x_{R 1} & y_{R 1} & z_{R 1}\end{array}$
Energia potencial total do sistema.
Energia potencial do corpo 1 .
Energia potencial do corpo 2 .
Energia potencial do rotor $R 1$.
Energia potencial do rotor $R 2$.
Tensão na armadura.
Tensão na armadura do motor 1 .
Tensão na armadura do motor 2 .
Tensão limite na armadura do motor 1 .
Tensão limite na armadura do motor 2 .
Vetor velocidade de um ponto $a$ com respeito a um corpo (ou referencial) B escrito em coordenadas de um sistema solidário a um referencial (ou corpo) $\mathrm{C}$.
Vetor ${ }_{\mathbf{B}}^{\mathbf{C}} \mathbf{v}_{\mathbf{a}}$ transposto.
Eixos do sistema de coordenadas cartesianas solidário ao corpo 0 .
Eixos do sistema de coordenadas cartesianas solidário ao corpo 1 .
Eixos do sistema de coordenadas cartesianas solidário ao corpo 2 .
Eixos do sistema de coordenadas cartesianas intermediário utilizado para obtenção do ângulo desejado para o corpo 2 .
Eixos do sistema de coordenadas cartesianas intermediário utilizado para obtenção do ângulo desejado para o corpo 2 .
Componentes do vetor posição do ponto $a$ com respeito ao ponto $\mathcal{O}$ escrito em coordenadas do sistema solidário ao referencial $\mathrm{G}$.
Componentes do vetor posição do alvo com respeito ao ponto $\mathcal{O}$ escrito em coordenadas do sistema solidário ao referencial $\mathrm{G}$.
Coordenadas do centróide do alvo no plano da imagem capturada pelo sensor de visão.
Eixos do sistema de coordenadas cartesianas solidário ao referencial inercial.
Eixos do sistema de coordenadas cartesianas solidário ao rotor $R 1$. 
$\begin{array}{lll}x_{R 2} & y_{R 2} & z_{R 2}\end{array}$

Z

Z.O.H.

Símbolos Gregos

$\alpha$

$\alpha^{\prime}$

$\alpha_{d}$

$\alpha_{m}$

$\bar{\alpha}$

$\beta$

$\beta$,

$\beta_{d}$

$\beta_{m}$

$\bar{\beta}$

$\gamma$

$\pm \Delta$

$\Delta t$

$\Delta t$

$\Delta t_{1}$

$\Delta t_{2}$

$\delta$

$\eta$

$\eta_{1}$

$\eta_{2}$

$\theta_{1}$

$\theta_{2}$

$\theta_{b}$
Eixos do sistema de coordenadas cartesianas solidário ao rotor $R 2$.

Zero.

Segurador de ordem zero ("zero order hold").

Ângulo de rotação do corpo 1 em relação ao corpo 0 .

Diferença entre $\alpha_{d}$ e $\alpha$.

Ângulo $\alpha$ desejado.

Ângulo de rotação do rotor $R 1$ em relação ao corpo 0 .

Valor do ângulo $\alpha$ obtido pelo encoder.

Ângulo de rotação do corpo 2 em relação ao corpo 1 .

Diferença entre $\beta_{d}$ e $\beta$.

Ângulo $\beta$ desejado.

Ângulo de rotação do rotor $R 2$ em relação ao corpo 1 .

Valor do ângulo $\beta$ obtido pelo encoder.

Ângulo de rolagem ("roll").

Incerteza de um sensor.

Atraso de tempo.

Atraso de tempo para atualização do sinal de controle quando se utiliza a arquitetura de controle do tipo 1 .

Atraso de tempo para atualização do sinal de saída das malhas externas nas arquiteturas de controle dos tipos 2 e 3 .

Atraso de tempo para atualização do sinal de controle (saída das malha internas) nas arquiteturas de controle dos tipos 2 e 3 .

Ângulo de inclinação ("pitch").

Metade da folga total em um redutor de velocidades.

Metade da folga total no redutor de velocidades utilizado entre o rotor $R l$ e o corpo 1 .

Metade da folga total no redutor de velocidades utilizado entre o rotor $R 2$ e o corpo 2 .

Deslocamento angular da extremidade esquerda do eixo sem inércia $(E)$ utilizado no modelo do redutor.

Deslocamento angular da extremidade direita do eixo sem inércia $(E)$ utilizado no modelo do redutor.

Ângulo $\theta_{2}$ menos ângulo $\theta_{\text {carga }}$. 


\begin{tabular}{|c|c|}
\hline$\theta_{b 1}$ & Ângulo $\theta_{b}$ do redutor entre o rotor $R 1$ e o corpo 1 . \\
\hline$\theta_{b 2}$ & Ângulo $\theta_{b}$ do redutor entre o rotor $R 2$ e o corpo 2 . \\
\hline$\theta_{\text {carga }}$ & Deslocamento angular da carga movimentada pelo motor. \\
\hline$\theta_{d}$ & Ângulo $\theta_{l}$ menos ângulo $\theta_{\text {carga }}$. \\
\hline$\theta_{d 1}$ & Ângulo $\theta_{d}$ do redutor entre o rotor $R l$ e o corpo 1 . \\
\hline$\theta_{d 2}$ & Ângulo $\theta_{d}$ do redutor entre o rotor $R 2$ e o corpo 2 . \\
\hline$\dot{\theta}_{i}$ & $\begin{array}{l}\text { Velocidade angular de um corpo } i \text { na direção do eixo de } \\
\text { rotação do corpo. }\end{array}$ \\
\hline$\dot{\theta}_{\text {residual }, i}$ & $\begin{array}{l}\text { Velocidade angular residual de um corpo } i \text { na direção do } \\
\text { eixo de rotação do corpo. }\end{array}$ \\
\hline$\theta_{\text {rotor }}$ & $\begin{array}{l}\text { Deslocamento angular do rotor, com respeito à carcaça do } \\
\text { motor, medido em torno do eixo axial do rotor. }\end{array}$ \\
\hline$\mu$ & $\begin{array}{l}\text { Grau de pertinência das funções de pertinência utilizadas no } \\
\text { FLC. }\end{array}$ \\
\hline$\mu_{i}$ & $\begin{array}{l}\text { Constante utilizada para o cálculo do coeficiente de } \\
\text { amortecimento numérico em um corpo } i \text {. }\end{array}$ \\
\hline$\xi$ & Valor real de uma variável. \\
\hline $\bar{\xi}$ & Valor de uma variável obtido por algum sensor. \\
\hline$\xi_{j}$ & Variável (coordenada) independente $j$. \\
\hline$\Xi_{j}$ & $\begin{array}{l}\text { Força (torque) generalizada não } \\
\text { correspondente à } \xi_{j} .\end{array}$ \\
\hline$\Xi_{\alpha}$ & $\begin{array}{l}\text { Força (torque) generalizada não } \\
\text { correspondente à variável independente } \alpha \text {. }\end{array}$ \\
\hline$\Xi_{\alpha_{m}}$ & $\begin{array}{l}\text { Força (torque) generalizada não } \\
\text { correspondente à variável independente } \alpha_{m} .\end{array}$ \\
\hline$\Xi_{\beta}$ & $\begin{array}{l}\text { Força (torque) generalizada não } \\
\text { correspondente à variável independente } \beta \text {. }\end{array}$ \\
\hline
\end{tabular}

$\begin{array}{ll}\Xi_{\beta_{m}} & \text { Força (torque) generalizada não conservativa } \\ & \text { correspondente à variável independente } \beta_{m} .\end{array}$
$\hat{\rho} \quad$ Ângulo de abertura.

$\hat{\rho}_{a z} \quad$ Ângulo de abertura em azimute do sensor de visão.

$\hat{\rho}_{e l} \quad$ Ângulo de abertura em elevação do sensor de visão.

$\sigma \quad$ Desvio-padrão.

$\psi \quad$ Ângulo de guinagem ("yaw”).

$\omega \quad$ Velocidade angular de um rotor, com respeito à carcaça do motor, na direção do eixo axial do rotor. 


\begin{tabular}{|c|c|}
\hline${ }_{\mathrm{B}}^{\mathrm{C}} \omega_{\mathrm{A}}$ & $\begin{array}{l}\text { Vetor velocidade angular de um corpo A com respeito a um } \\
\text { corpo (ou referencial) B escrito em coordenadas de um } \\
\text { sistema solidário a um referencial (ou corpo) C. }\end{array}$ \\
\hline${ }_{B}^{C} \omega_{A}^{T}$ & Vetor ${ }_{\mathbf{B}}^{\mathbf{C}} \boldsymbol{\omega}_{\mathbf{A}}$ transposto. \\
\hline$\omega d_{x_{2}}$ & $\begin{array}{l}\text { Componente da velocidade angular inercial desejada do } \\
\text { corpo } 2 \text { na direção } x_{2} \text {. }\end{array}$ \\
\hline$\omega d_{z_{2}}$ & $\begin{array}{l}\text { Componente da velocidade angular inercial desejada do } \\
\text { corpo } 2 \text { na direção } z_{2} \text {. }\end{array}$ \\
\hline$\omega d_{i n c}$ & $\begin{array}{l}\text { Incremento para a componente da velocidade angular } \\
\text { inercial desejada. }\end{array}$ \\
\hline$\omega d_{i n c_{M}}$ & $\begin{array}{l}\text { Constante utilizada para normalização das funções de } \\
\text { pertinência de saída empregadas no FLC. }\end{array}$ \\
\hline$\omega d_{i n c x_{2}}$ & $\begin{array}{l}\text { Incremento para a componente da velocidade angular } \\
\text { inercial desejada do corpo } 2 \text { na direção } x_{2} \text {. }\end{array}$ \\
\hline$\omega d_{i n z_{2}}$ & $\begin{array}{l}\text { Incremento para a componente da velocidade angular } \\
\text { inercial desejada do corpo } 2 \text { na direção } z_{2} \text {. }\end{array}$ \\
\hline$\omega_{\min , 1}$ & $\begin{array}{l}\text { Velocidade angular (do corpo } 1 \text { com respeito ao corpo } 0 \text { ) } \\
\text { de transição entre os regimes de "stick" e "slip". }\end{array}$ \\
\hline$\omega_{\min , 2}$ & $\begin{array}{l}\text { Velocidade angular (do corpo } 2 \text { com respeito ao corpo 1) } \\
\text { de transição entre os regimes de "stick" e "slip". }\end{array}$ \\
\hline$\omega_{\min , i}$ & $\begin{array}{l}\text { Velocidade angular de transição entre os regimes de "stick" } \\
\text { e "slip" na direção do eixo de rotação do corpo. }\end{array}$ \\
\hline$\omega_{\min , R l}$ & $\begin{array}{l}\text { Velocidade angular (do rotor } R 1 \text { com respeito ao corpo } 0 \text { ) } \\
\text { de transição entre os regimes de "stick" e "slip". }\end{array}$ \\
\hline$\omega_{\min , R 2}$ & $\begin{array}{l}\text { Velocidade angular (do rotor } R 2 \text { com respeito ao corpo } 1 \text { ) } \\
\text { de transição entre os regimes de "stick" e "slip". }\end{array}$ \\
\hline$\omega_{x_{2}}$ & $\begin{array}{l}\text { Componente da velocidade angular inercial do corpo } 2 \text { na } \\
\text { direção } x_{2} \text {. }\end{array}$ \\
\hline$\omega_{z_{2}}$ & $\begin{array}{l}\text { Componente da velocidade angular inercial do corpo } 2 \text { na } \\
\text { direção } z_{2} \text {. }\end{array}$ \\
\hline$\Omega$ & $\begin{array}{l}\text { Velocidade angular do movimento circulatório do alvo em } \\
\text { um plano paralelo ao plano formado pelos eixos } x_{G} \text { e } z_{G} \text {. }\end{array}$ \\
\hline
\end{tabular}

